\title{
Influencia del modelo de Educación Deportiva en las necesidades psicológicas básicas
}

\section{Influence of the Sport Education Model in the psychological basic needs}

\author{
Influência do Modelo de Educação Esportiva nas \\ necessidades psicológicas básicas
}

\author{
Ricardo Cuevas, Luis M. García-López y Onofre Contreras
}

Universidad de Castilla-La Mancha

Resumen: Tomando la teoría de la Autodeterminación como marco, el objetivo del estudio fue conocer el impacto del modelo de Educación Deportiva en la satisfacción de las necesidades psicológicas básicas en alumnos de Educación Secundaria en Educación Física. Para ello se seleccionaron dos grupos de alumnos de entre 15 y 17 años: un grupo experimental $(n=43)$ que desarrolló un programa de intervención basado en el modelo de Educación Deportiva de 19 sesiones, y un grupo de control $(n=43)$ que cursó un programa de 19 sesiones de Educación Física tradicional. Se tomaron medidas pre- $y$ pos-test en ambos grupos. Los resultados hallaron mejoras significativas en la satisfacción de competencia en los alumnos que utilizaron el modelo de Educación Deportiva. Se discutieron los resultados obtenidos subrayando la idoneidad del modelo de Educación Deportiva para favorecer la satisfacción de las necesidades psicológicas básicas en Educación Física. Palabras clave: autodeterminación, programa de intervención, adolescentes, deporte, actividad física.

Abstract: Using the Self-determination Theory as a guide framework, the aim of the study was to analyze the impact of Sport Education Model in psychological basic need satisfaction in Physical Education secondary students. Two groups were used for the study: an experimental group $(N=43)$, which received 19 lessons following the Sport Education Model, and a control group $(N=43)$, which received 19 traditional Physical Education lessons. Pre- and post-intervention measures were taken in both groups. The results showed significant improvements in the satisfaction of the competence in the Sport Education group. The results were discussed and it was emphasized the suitability of the Sport Education Model to improve the satisfaction of psychological basic needs in Physical Education.

Key words: self-determination, intervention program, adolescents, sport, physical activity.

Resumo: Usando a teoria da Autodeterminação (Deci \& Ryan, 1985; Ryan \& Deci, 2002) como referência, o objetivo do estudo foi analisar o impacto do modelo de Educação Esportiva em satisfação psicológica necessidade básica em alunos do ensino secundário de Educaçăo Física. Dois grupos foram utilizados para o estudo: um grupo experimental $(N=43)$, que recebeu 19 liçốes seguindo a modelo de Educaçấo Esportiva, e um grupo controle $(\mathrm{N}=43)$, que recebeu 19 liçóes de Educação Física tradicionais. Medidas de pré-e pós-intervençâo foram tomadas em ambos os grupos. Os resultados mostraram melhorias significativas na satisfação da competência do grupo Esporte Educaçáo. Os resultados foram discutidos e enfatizou-se a adequação do modelo de Educação Esportiva para melhorar a satisfação das necessidades básicas psicológicos em Educação Física.

Palavras chave: auto-determinação, programa de intervençấo, adolescentes, esportes, atividade física.

\section{Introducción}

La satisfacción de las necesidades psicológicas básicas de los alumnos constituye un factor psicológico relevante para el rendimiento académico, la autorregulación del aprendizaje y el bienestar (Niemiec y Ryan, 2009). En este sentido, la satisfacción de la competencia, la autonomía y la relación en clase de Educación Física puede resultar determinante para favorecer la consecución de ciertos objetivos de esta asignatura, tales como el fomento de la práctica regular de actividad física o el aprendizaje deportivo. En esta línea, varios estudios han constatado que propuestas didácticas basadas en el modelo de Educación Deportiva (Siedentop, 1994) favorecen

\footnotetext{
Dirección para correspondencia [Correspondence address]: Dr. Ricardo Cuevas. Plaza de la Universidad, 3. C.P. 02071. Facultad Educación. Universidad Castilla-La Mancha. Albacete (Espańa). E-mail: ricardo. cuevas@uclm.es
}

el desarrollo personal y social (Kinchin, Wardle, Roderick, y Sprosen, 2004), necesidades psicológicas básicas como la relación (Perlman, 2010, 2011), o la motivación autodeterminada (Perlman, 2011) en alumnos de educación secundaria. En esta línea, en el presente trabajo se pretende comparar el impacto del modelo de Educación Deportiva y del modelo tradicional de Educación Física en la satisfacción de las necesidades psicológicas básicas de un grupo de estudiantes adolescentes.

En este contexto, la teoría de la Autodeterminación (Deci y Ryan, 1985; Ryan y Deci, 2002) resulta útil para entender los procesos motivacionales. Esta macro-teoría ofrece un amplio marco para estudiar la motivación, el desarrollo personal y el bienestar, subrayando la importancia que posee el entorno y los factores sociales en la iniciativa y el com- 
portamiento de las personas. Dentro de ésta se formula la micro-teoría de las Necesidades Psicológicas Básicas (Deci y Ryan, 2000), que señala la existencia de tres necesidades psicológicas básicas (competencia, autonomía y relación) que han de ser satisfechas para garantizar el buen funcionamiento y la salud psicológica de los individuos. El concepto de competencia se refiere al nivel de maestría y eficacia que siente el sujeto al realizar una tarea. La idea de autonomía representa el sentimiento de control que posee la persona sobre su propio comportamiento. Por último, la necesidad de relación constituye el sentimiento de conexión con el grupo de sujetos que le rodean. A través de estudios transversales en el contexto de la educación física en adolescentes, la satisfacción de estas necesidades se ha asociado a numerosos beneficios como mayor motivación autodeterminada, concentración, persistencia, afecto positivo o bienestar (Ntoumanis, 2005; Standage, Duda y Ntoumanis, 2005).

El modelo de Educación Deportiva trata de introducir al alumnado en una experiencia real del deporte (Siedentop, Hastie y van der Mars, 2011). Para ello, establece una serie de elementos propios del deporte que deben formar parte de las unidades de enseñanza del mismo en el contexto escolar. Estos son la estructuración de las unidades didácticas en fases de pretemporada, competición formal y fases finales; la formación de equipos estables; el registro de datos; y la festividad (Siedentop et al., 2011). A esto se une la utilización de los diferentes roles que se pueden encontrar en el deporte (entrenador, árbitro, preparador físico,...), los cuales permiten al alumno vivir el deporte desde las diferentes perspectivas posibles, enriqueciendo su conocimiento y entendiendo las diferentes problemáticas que supone para cada rol.

Varios estudios han constatado la influencia del modelo de Educación Deportiva en el desarrollo positivo del alumnado (ver Hastie, Martínez de Ojeda y Calderón-Luquin, 2011; Wallhead y O'Sullivan, 2005). En este sentido, Sinelnikov, Hastie y Prusak (2007) constataron a través de un estudio cuantitativo y cuasi-experimental que el modelo de Educación Deportiva puede ayudar a favorecer un comportamiento más autodeterminado en estudiantes adolescentes de Educación Física. Sus características y formas de organización favorecen la creación de un ambiente en clase de apoyo de las necesidades psicológicas básicas. Con respecto a la competencia, el modelo de Educación Deportiva está centrado en la promoción del éxito motor, cognitivo y afectivo del alumno (Siedentop, 1994). En esta línea, son varios los trabajos que han confirmado una mejora de las percepciones de competencia y éxito asociadas al modelo de Educación Deportiva (MacPhail, Gorely, Kirk y Kinchin, 2008; Spittle y Byrne, 2009) en escolares. La relación también se ve reforzada en el modelo de Educación Deportiva al aumentar, con respecto a la enseńanza deportiva tradicional, el tiempo y las oportunidades de interacción entre los estudiantes, favoreciendo así su socialización (Carlson y Hastie, 1997). Por último, el modelo de Educación Deportiva se basa en un enfoque de apoyo a la autonomía del alumno (Wallhead, Hagger y Smith, 2010). Así, hay estudios que confirman una mejora de las percepciones de autonomía y de oportunidades de elección en clases basadas en el modelo de Educación Deportiva (MacPhail et al., 2008; Siedentop et al., 2011).

Por otra parte, diversos estudios han constatado que el modelo tradicional de enseńanza deportiva no se asocia con el apoyo a las necesidades psicológicas básicas. Así, trabajos de corte cuasi-experimental en el contexto de la educación física (Perlman, 2011; Wallhead y Ntoumanis, 2004), constataron que intervenciones basadas en el modelo tradicional no tenían un impacto positivo en las percepciones de competencia, autonomía o relación del alumnado adolescente.

Han sido varios los trabajos que han avanzado en el estudio de los procesos motivacionales y de bienestar psicológico en relación con el modelo de Educación Deportiva. Así, se ha comprobado que éste favorece la mejora de la percepción de apoyo en clase a las necesidades psicológicas básicas (Perlman, 2011), así como de la competencia percibida (Spittle y Byrne, 2009). No obstante, continúan existiendo limitaciones que sugieren seguir investigando para ampliar los conocimientos de esta línea (Hastie et al., 2011). En este sentido, resulta de especial interés clarificar el impacto del modelo de Educación Deportiva en las percepciones de relación y autonomía, ya que existen trabajos (Brock, Rovengo, y Oliver, 2009) que sugieren la posible influencia negativa en dichas variables. Desde un planteamiento cercano a estos trabajos, el presente estudio pretende conocer el impacto de un programa basado en el modelo de Educación Deportiva en alumnos de Educación Física con respecto a la satisfacción las tres necesidades psicológicas básicas. Para ello se plantea la siguiente hipótesis: el programa basado en el modelo de Educación Deportiva mejorará la significativamente la satisfacción de las tres necesidades psicológicas básicas de los estudiantes del grupo experimental. En la misma línea, el programa de educación física tradicional mantendrá los mismos niveles de satisfacción de las necesidades psicológicas básicas de los alumnos del grupo de control antes y después de la intervención.

\section{Método}

\section{Participantes}

Los participantes fueron 86 estudiantes, 49 chicas (57\%) y 37 chicos (43\%), con una edad entre 15 y 17 años $(M$ edad $=$ 15.65; $D T=.78$ ) y que cursaban Educación Física en cuarto curso de Educación Secundaria Obligatoria en dos centros de Castilla-La Mancha. Se realizó un muestreo aleatorio por conglomerados (Azorín y Sánchez-Crespo, 1994), seleccionando un total de cuatro grupos de alumnos. La facilidad de 
acceso a dichos centros educativos fue un factor determinante para la selección de la muestra.

\section{Diseño}

Se utilizó un diseño cuasi-experimental con medidas pre- y post-test en el grupo experimental y en el de control, a los cuales fueron asignados tratamientos diferentes. Debido al uso de grupos ya establecidos en clases, no fue posible realizar una asignación totalmente al azar de los participantes según las dimensiones de la variable independiente (modelo de Educación Deportiva y Educación Física tradicional). Así, se realizó una asignación al azar de dos de los grupos $(n=43)$ como grupo experimental, y los otros dos formaron el grupo de control $(n=43)$. En este sentido, se utilizó un diseño de grupo de control no equivalente (Campbell y Stanley, 1963), lo que implica que a priori los grupos pueden ser desiguales debido a la previa configuración de las clases.

\section{Procedimiento}

Se informó a los centros y a los padres y madres, así como a los estudiantes del objetivo del trabajo, y se lograron los permisos y consentimientos necesarios (Faden, Beauchamp, y King, 1986). Seguidamente, se procedió con la intervención y la recogida de datos. La investigación cumplió con todos los requerimientos éticos, respetando el anonimato y la voluntariedad de los participantes. El estudio se llevó a cabo en cuatro fases. Primero se llevó a cabo el diseño de los programas de intervención para ambos grupos. En segundo lugar, se realizó un pre-test en ambos grupos. A continuación, el programa basado en el modelo de Educación Deportiva se aplicó al grupo experimental, mientras que el de control desarrolló sus clases de Educación Física siguiendo el modelo tradicional. Finalizada la intervención, se realizó otra medida pos-test en los dos grupos. Los cuestionarios, tanto en el pre-test como en el post-test, fueron completados en clase de Educación Física en un tiempo aproximado de 10 minutos. Un investigador expuso las instrucciones para su cumplimentación y aclaró las dudas de los participantes.

\section{Diseño y validación de los programas de intervención}

Los investigadores y el docente encargado del grupo experimental desarrollaron un programa siguiendo la estructura del modelo de Educación Deportiva (Siedentop et al., 2011) para la enseñanza del voleibol. El diseño del programa se realizó en base a las indicaciones de Moreno y Martínez (2006) para favorecer el apoyo a las necesidades psicológicas básicas. La intervención consistió en una unidad didáctica de voleibol con una duración de 19 sesiones de 55 minutos cada una (dos por semana, a lo largo de dos meses y medio). La duración total del tratamiento se consideró suficiente para estudiar los posibles efectos en las variables dependientes en base a anteriores trabajos que utilizaron una temporalización similar (García-López y Gutiérrez, 2013; Hastie y Sinelnikov, 2010). El programa se desarrolló durante el horario habitual de la asignatura de Educación Física.

Los investigadores y el profesor establecieron una serie de pautas que se reflejaron en el diseńo del programa: cinco equipos por clase y cinco roles para cada equipo (capitánentrenador, preparador físico, árbitro, responsable de estadísticas e informes y miembro del comité de disciplina y organización). La formación de dichos equipos se realizó en base a un sorteo y la asignación de roles fue decidida libremente por parte de los miembros de los equipos. Para aumentar la fidelidad con respecto al modelo de Educación Deportiva, el programa utilizó los mismos materiales curriculares (portfolios, fichas e informes) utilizados previamente en anteriores estudios (García-López y Gutiérrez, 2013).

Con respecto a la secuencia de las sesiones del programa (Tabla 1), las primeras siete se dedicaron a presentar el modelo de Educación Deportiva y los roles, la elaboración de los equipos, el aprendizaje de elementos técnico-tácticos y la preparación de la competición. Estas sesiones de entrenamiento comenzaron con un calentamiento de 10 minutos, seguido de 40 minutos de actividades y juegos para el aprendizaje del voleibol, y para finalizar una serie de estiramientos durante cinco minutos. A lo largo de dichas sesiones, los alumnos fueron asumiendo sus roles y participando progresivamente en la toma de decisiones en clase. Por ejemplo, el capitán-entrenador diseñó y dirigió, con las orientaciones y el apoyo del docente, las sesiones de entrenamiento técnico-táctico de su equipo. En las siguientes ocho sesiones se desarrolló la competición regular, dejando tres de ellas intercaladas para entrenamiento. Cada sesión de competición se compuso del calentamiento de 10 minutos, de dos partidos durante los siguientes 35 minutos y de los estiramientos y la cumplimentación de los informes en los últimos 10 minutos de clase. Los árbitros de los equipos a los que no les correspondía competir se encargaron de arbitrar dichos encuentros, mientras que el resto de estudiantes asumió el rol de público. Los partidos se jugaron en situación reducida de tres jugadores contra tres, con la obligatoriedad de hacer cambios para que todos los miembros del equipo participasen equitativamente en la competición. En las últimas cuatro sesiones se celebraron las fases finales y el evento festivo, en el que se el comité organizador entregó diplomas tanto a los ganadores como a todos los participantes.

El profesor encargado de este programa tenía más de diez años de experiencia y recibió formación y entrenamiento previo a tal efecto. En este sentido, se desarrolló un seminario de 12 sesiones de una hora en el cual se abordaron aspectos teóricos y prácticos del modelo de Educación Deportiva. Un investigador externo evaluó la correcta aplicación del programa en el grupo experimental, siguiendo para ello la hoja de 
registro observacional de Ko, Wallhead, y Ward (2006), utilizada en anteriores investigaciones (García-López y Gutiérrez, 2013; Sinelnikov, 2009). El profesor que llevó a cabo la intervención cumplió todos los indicadores de la hoja de registro.

Paralelamente, los investigadores junto con el docente encargado de impartir docencia al grupo de control, diseñaron otro programa para el aprendizaje del voleibol (Tabla 1) basado en estilos de enseńanza tradicionales (Sicilia y Delgado, 2002). La duración de esta intervención fue también de 19 sesiones de 55 minutos, dedicando las 16 primeras a la enseñanza de elementos técnico-tácticos del voleibol (toque de dedos, toque de mano baja, remate o saque, entre otros) a través de estilos de enseñanza como el mando directo o la asignación de tareas. Estas sesiones comenzaron por un calentamiento de 10 minutos, seguido de explicaciones y prácticas sobre el voleibol durante 40 minutos, y finalizando con estiramientos de cinco minutos. Todas las actividades de dichas sesiones fueron dirigidas exclusivamente por el docente. Las tres últimas sesiones se dedicaron a competición de equipos de seis alumnos contra seis seleccionados y organizados por el profesor. Un investigador externo analizó la correcta aplicación de dicho programa en base a los criterios señalados por Contreras (1998) para la utilización de estilos de enseñanza tradicionales. El profesor encargado del impartir docencia al grupo de control cumplió con todos los criterios.

Tabla 1. Principales contenidos y actividades de los programas de intervención por sesiones.

\begin{tabular}{|c|c|c|}
\hline Sesión & Modelo de Educación Deportiva & Modelo Tradicional \\
\hline 1 & Introducción y formación de equipos. Elección de capitán-entrenador. & Introducción. Juego voleibol $6 \times 6$. \\
\hline 2 & Nombres equipos y otros roles: preparador físico. Saque de seguridad y toque de dedos. & Toque de dedos $1 \times 1$. \\
\hline 3 & Rol de árbitro. Saque de tenis y toque de dedos. & Toque de dedos $1 \times 1$. \\
\hline 4 & Presentación de escudos y camisetas. Toque de dedos y de mano baja. Táctica. & Toque de dedos $2 \times 2$. \\
\hline 5 & Rol comité disciplina y organización. Toque mano baja, toque de dedos y remate. Táctica. & Toque de dedos $2 \times 2$. \\
\hline 6 & Rol de estadístico. Sorteo y calendario del campeonato. Remate y bloqueo. Táctica. & Toque de mano baja $1 \mathrm{x} 1$. \\
\hline 7 & Entrenamiento para el campeonato. & Toque de mano baja $1 \times 1$. \\
\hline 8 & Competición (fase regular). & Toque de dedos y de mano baja. \\
\hline 9 & Entrenamiento para el campeonato. & Saque de seguridad. \\
\hline 10 & Competición (fase regular). & Saque de tenis. \\
\hline 11 & Entrenamiento para el campeonato. & Remate y bloqueo. \\
\hline 12 & Competición (fase regular). & Remate y bloqueo. \\
\hline 13 & Entrenamiento para el campeonato. & Táctica de ataque $6 \times 6$. \\
\hline 14 & Competición (fase regular). & Táctica de ataque $6 \times 6$. \\
\hline 15 & Competición (fase regular). & Táctica de defensa $6 \times 6$. \\
\hline 16 & Competición (semifinal 1) & Táctica de defensa $6 \times 6$. \\
\hline 17 & Competición (semifinal 2) & Competición 6x6. \\
\hline 18 & Competición (final del grupo-clase). & Competición 6x6. \\
\hline 19 & Competición (final de entre clases) y evento festivo. Entrega de diplomas. & Competición 6x6. \\
\hline
\end{tabular}

\section{Instrumentos}

Satisfacción de las necesidades psicológicas básicas. Se usó la versión en español (Moreno, González-Cutre, Chillón y Parra, 2008) de la Basic Psychological Needs in Exercise Scale (BPNES; Vlachopoulos y Michailidou, 2006). El encabezado "en mis clases de educación física” fue seguido de 12 ítems (cuatro por factor) para evaluar la satisfacción de la autonomía (e. g. "la forma de realizar los ejercicios coincide perfectamente con la forma en que yo quiero hacerlos"), la competencia (e. g. "siento que he tenido una gran progresión con respecto al objetivo final que me he propuesto") y la relación (e. g. "me siento muy cómodo cuando hago los ejercicios con los demás compañeros"). Las respuestas oscilaron del uno (totalmente en desacuerdo) al cinco (totalmente de acuerdo). Moreno, Cervelló, Montero y Vera (2012) obtuvieron adecuados índices psicométricos de esta versión del instrumento. La adaptación al español del instrumento (Moreno et al., 2008) obtuvo unos valores de ajuste aceptables en validez de constructo $\left(\chi^{2} / g . l .=3.29 ; \mathrm{CFI}=.94 ; \mathrm{IFI}=.94 ; \mathrm{TLI}=.92 ; \mathrm{RMSEA}\right.$ $=.07$; SRMR $=.07)$; buena fiabilidad interna, con alfas de Cronbach de .81 para la autonomía, .78 para la competencia y .84 para la relación; y adecuada fiabilidad test-retest. 


\section{Análisis de datos}

Se utilizó el paquete estadístico IBM-SPSS 19.0. Se calculó la fiabilidad de las escalas a través del alfa de Cronbach para el pre- y el post-test. Asimismo, se procedió a calcular las medias y las desviaciones típicas para ambas mediciones. En línea con anteriores trabajos que utilizaron diseños similares en contextos educativos (Papaioannou, Evaggelinou, Barkoukis, y Block, 2013; Perlman, 2010; Wallhead y Ntoumanis, 2004), se realizó un ANOVA de medidas repetidas para cada variable dependiente, con el objeto de comprobar si la aplicación del programa tuvo efectos significativos. Se tomó como variable intra-sujetos el tiempo (pre-y post-test) y como variable inter-sujetos el grupo (experimental y control). Asimismo se aplicó la prueba de esfericidad de Mauchly obteniendo resultados no significativos, por lo que se asumió la esfericidad de los datos (Winer, Brown, y Michels, 1991). Para prevenir el error tipo I al interpretar los resultados de este tipo de análisis se utilizó el método de ajuste de Bonferroni que, para este caso, fija un nivel de significación de $p \leq .0125$ al compararse cuatro mediciones en el análisis (Ntoumanis, 2001). Además de analizar la significatividad de los cambios, se halló el tamaño del efecto a través del eta-cuadrado parcial para cada variable. Olejnik y Algina (2000) seńalaron la importancia de calcular este estadístico al comparar variables entre grupos, especialmente cuando el número de sujetos que componen la muestra no es elevado.

\section{Resultados}

Los alfas de Cronbach (Tabla 2) informan de valores de fiabilidad adecuados de las variables (Hair, Anderson, Tatham y Black, 1998). También en dicha tabla se recogen los valores medios y las desviaciones típicas, pudiendo observar algunos de los cambios producidos desde la primera a la segunda medición en ambos grupos.

Tabla 2. Medidas, desviaciones típicas y alfas de Cronbach de las medidas pre- y post-test en los grupos experimental y control.

\begin{tabular}{|c|c|c|c|c|c|c|c|c|c|c|}
\hline & \multicolumn{5}{|c|}{ Pre-test } & \multicolumn{5}{|c|}{ Post-test } \\
\hline & \multirow[b]{2}{*}{$\alpha$} & \multicolumn{2}{|c|}{ Experimental } & \multicolumn{2}{|c|}{ Control } & \multirow[b]{2}{*}{$\alpha$} & \multicolumn{2}{|c|}{ Experimental } & \multicolumn{2}{|c|}{ Control } \\
\hline & & $M$ & $D T$ & $M$ & $D T$ & & $M$ & $D T$ & $M$ & DT \\
\hline Competencia & ,75 & 3,75 &, 85 & 3,44 &, 80 & ,76 & 4,12 &, 71 & 3,14 &, 75 \\
\hline Autonomía &, 84 & 3,44 &, 86 & 2,94 &, 84 &, 82 & 3,84 &, 73 & 2,96 &, 86 \\
\hline Relación & ,83 & 4,31 &, 67 & 3,53 & ,98 &, 85 & 4,57 &, 48 & 3,34 & ,92 \\
\hline
\end{tabular}

La Tabla 3 recoge los resultados del ANOVA 2x2. En lo que respecta a la interacción de los efectos (tiempo x grupo), los resultados mostraron modificaciones significativas para la satisfacción de la competencia, lo que revela que el programa basado en el modelo de Educación Deportiva produjo mejoras a considerar en la percepción de competencia $(p=.012)$ de los participantes del grupo experimental. La mejora en esta variable también se constata a través del considerable valor del tamaño del efecto encontrado $\left(\eta 2_{\text {parcial }}=.073\right)$. Por otro lado, a pesar de que los cambios no fueron significativos, se pudieron constatar ligeras mejoras en el grupo experimental tanto en la satisfacción de la autonomía $\left(\eta 2_{\text {parcial }}=.027\right)$ y como en la de la relación $\left(\eta 2_{\text {parcial }}=.034\right)$.

Tabla 3. Efectos del tiempo y de la interacción de los efectos de tiempo y grupo en los ANOVAS $2 \times 2$.

\begin{tabular}{lllllllll}
\hline & \multicolumn{3}{l}{ Tiempo } & & \multicolumn{3}{c}{ Tiempo * grupo } \\
\cline { 2 - 3 } \cline { 7 - 8 } & $F$ & $p$ & $\eta 2_{\text {parcial }}$ & & $F$ & $p$ & $\eta 2_{\text {parcial }}$ \\
Competencia & 074 &, 787 &, 001 & & 6,635 &, 012 &, 073 \\
Autonomía & 2,821 &, 097 &, 032 & & 2,370 &, 127 &, 027 \\
Relación &, 079 &, 779 &, 001 & & 2,922 &, 091 &, 034 \\
\hline
\end{tabular}

\section{Discusión}

El estudio pretendió averiguar, desde la perspectiva de la teoría de la Autodeterminación (Deci y Ryan, 1985; Ryan y Deci, 2002), el impacto de un programa basado en el modelo de Educación Deportiva, en comparación con el modelo tradicional, en la satisfacción de las necesidades psicológicas básicas en estudiantes de Educación Física. En términos generales, el impacto de dicho programa sobre el alumnado fue positivo. No obstante, la hipótesis planteada se confirmó parcialmente a través de la mejora significativa de la satisfacción de competencia. Este resultado está en línea con otras investigaciones que hallaron un incremento en la percepción de competencia del alumnado que cursó el modelo de Educación Deportiva, como el trabajo de Spittle y Byrne (2009). Estos autores compararon a través de un estudio cuasi-experimental el modelo de Educación Deportiva con el modelo tradicional en estudiantes de Educación Física en Educación Secundaria. En este sentido, constataron mejoras significativas de la percepción de competencia del alumnado que cursó el modelo de Educación Deportiva. Este hallazgo también coincide con lo apuntado por Gutiérrez, García-López, Hastie y Calderón-Luquin (2013), que confirmaron mejoras en 
la percepción de competencia de alumnos de Educación Primaria y Secundaria que utilizaron el modelo de Educación Deportiva. Esto podría deberse a que el modelo de Educación Deportiva está diseñado originalmente para favorecer la práctica y el aprendizaje deportivo de los estudiantes (Siedentop, 1994). En esta línea, tal y como han señalado varios trabajos (Hastie, Sinelnikov, Wallhead y Layne, 2014; Wallhead y Ntoumanis, 2004) el modelo de Educación Deportiva fomenta un clima motivacional de clase orientado hacia la tarea, circunstancia relacionada con la mejora de la percepción de competencia del alumnado (Elliot y Conroy, 2005; Moller y Elliot, 2006). Así, estudios previos han asociado el modelo de Educación Deportiva en el contexto educativo a mejoras en el conocimiento táctico (Browne, Carlson y Hastie, 2004), en la comprensión táctica (Clarke y Quill, 2003), en el rendimiento (Hastie, 1998; Hastie y Trost, 2002; Hastie, Sinelnikov y Guarino, 2009; Pritchard, Hawkins, Wiegand, y Metzler, 2008) y en el aprendizaje percibido de los escolares (Browne et al., 2004). Asimismo, el modelo de Educación Deportiva ofrece a los alumnos la posibilidad de seleccionar diversos roles dentro de un mismo equipo. Este hecho facilita que el estudiante pueda escoger un rol ajustado a sus propias fortalezas personales, lo que favorece a su vez la ejecución exitosa y responsable de sus tareas en clase (Hastie, 1996) y, en consecuencia, la posible mejora de su percepción de competencia.

La satisfacción de la relación y la autonomía también registraron pequeñas mejoras en el grupo experimental, aunque no significativas. Este hecho contrasta con los resultados de otros trabajos que confirmaron que el modelo de Educación Deportiva podría favorecer importantes mejoras tanto en la autonomía (MacPhail et al., 2008; Siedentop et al., 2011), como las relaciones sociales (Carlson y Hastie, 1997; Perlman, 2010) del alumnado. Para interpretar estos resultados resultan de interés los hallazgos de Brock et al. (2009), que a través de un trabajo cualitativo observaron que los diferentes estatus sociales podían influir en las interacciones de los grupos en las sesiones. En este sentido, apuntaron que los alumnos con alto estatus social podrían cohibir las opiniones y comportamientos otros estudiantes más introvertidos. En este caso, las percepciones de relación y autonomía de ciertos alumnos podrían verse limitadas.

En cuanto a los resultados referidos a la intervención con el modelo tradicional de enseńanza deportiva, basado en estilos de enseńanza más controladores, hay que señalar que no encuentran modificaciones en la satisfacción de las necesidades psicológicas básicas, en línea con lo planteado en la hipótesis y con los resultados de anteriores trabajos (Perlman, 2011; Wallhead y Ntoumanis, 2004).

A pesar del notable interés del estudio, éste presenta una serie de limitaciones. El número de participantes que componen la muestra y su selección, así como la duración de la intervención, obligan a ser cautelosos con la generalización de conclusiones. En este sentido, sería de provecho el planteamiento de trabajos experimentales de mayor duración y con más participantes en aras a obtener resultados más concluyentes. Este tipo de trabajos podrían ayudar a confirmar o refutar las tendencias de mejora que se han hallado en este trabajo con respecto a la satisfacción de la autonomía y la relación.

Además, los resultados apuntan a nuevas cuestiones a resolver en el ámbito del modelo de Educación Deportiva. En primer lugar, en línea con lo apuntado por Brock et al. (2009), sería de interés aclarar si las interacciones dentro de cada equipo pueden verse afectadas por la desigualdad de estatus social de los alumnos en clase, y si esto afecta a la satisfacción de necesidades psicológicas básicas como la autonomía o la relación. Asimismo, los modelos de apoyo a la autonomía del alumno, como el de Educación Deportiva, poseen potencial para facilitar la transferencia de motivación y práctica físicodeportiva de Educación Física a otros contextos de carácter extracurricular (Wallhead et al., 2010; Wallhead, Garn, y Vidoni, 2013). En este sentido, es importante señalar que la percepción de competencia del alumno (variable que ha mejorado significativamente con el modelo de Educación Deportiva en este trabajo) representa uno de los constructos que más influyen en la intención de practicar actividad física (Cuevas, Contreras, Fernández y González-Martí, 2014) al terminar la escolarización obligatoria. Por ende, sería de interés seguir ampliando el conocimiento acerca del impacto que el modelo de Educación Deportiva podría tener en el fomento de la práctica deportiva más allá de la etapa escolar.

\section{Aplicaciones prácticas}

Trabajos como el actual señalan que el uso del modelo de Educación Deportiva puede favorecer la satisfacción de las necesidades psicológicas básicas de los escolares, como es el caso de la competencia. Por el contrario, los métodos tradicionales y controladores no evidencian influencia sobre las mismas. Así, teniendo en cuenta que la satisfacción de las necesidades psicológicas básicas favorece el bienestar y la motivación autodeterminada en el alumnado (Vallerand, 2007), se sugiere la aplicación del modelo de Educación Deportiva a la hora tratar los contenidos referidos a deporte en clase de Educación Física en Educación Secundaria. De este modo se favorecerá el bienestar y la motivación autodeterminada del alumnado. En esta línea, para la aplicación práctica del modelo de Educación Deportiva, se plantea que el docente vigile especialmente el cumplimiento de normas que favorecen la igualdad dentro de los equipos y que se contemplan dentro del modelo (p. e. mismo tiempo de participación activa de todos los componentes del equipo en las competiciones), para evitar que la autonomía o la relación de ciertos alumnos se vea limitada por la acción de otros miembros de su equipo. 


\section{Referencias}

1. Azorín, F. y Sánchez-Crespo, J. L. (1994). Métodos y aplicaciones del muestreo. Madrid: Alianza.

2. Brock, S. J., Rovengo, I., y Oliver, K. L. (2009). The influence of student status on student interactions and experiences during a sport education unit. Physical Education and Sport Pedagogy, 14(4), 355-375.

3. Browne, T.B.J., Carlson, T. B, y Hastie. P. A. (2004). A comparison of rugby seasons presented in traditional and sport education formats. European Physical Education Review, 10(2), 192-214. DOI: 10.1177/1356336X04044071.

4. Campbell, D. T. y Stanley, J. C. (1963). Experimental and quasiexperimental designs for research. Reprinted from N. L. Gage (Ed.), Handbook of research on teaching. Boston: Rand McNally.

5. Carlson, T.B. y Hastie, P. (1997). The student social system within sport education. Journal of Teaching in Physical Education, 16(2), 176195.

6. Clarke, G. y Quill, M. (2003). Researching sport education in action: a case study. European Physical Education Review, 9(3), 253-266.

7. Conteras, O. (1998). Didáctica de la educación física: un enfoque constructivista. Barcelona: Inde.

8. Cuevas, R., Contreras, O., Fernández, J. G., y González-Martí, I. (2014). Influencia de la motivación y el autoconcepto físico sobre la intención de ser físicamente activo. Revista Mexicana de Psicología, 31(1), 17-24.

9. Deci, E. L. y Ryan, R. M. (1985). Intrinsic motivation and self-determination in human behavior. New York: Academic Press.

10. Deci, E.L. y Ryan, R.M. (2000). The "what" and "why" of goal pursuits: Human needs and the self-determination of behaviour. Psychological Inquiry, 11, 227-268.

11. Elliot, A. J. y Conroy, D. E. (2005). Beyond the dichotomous model of achievement goals in sport and exercise psychology. Sport and Exercise Psychology Review, 1(1), 17-25.

12. Faden, R.R., Beauchamp, T.L., y King, N.N. (1986). A history and theory of informed consent. Oxford, UK: Oxford University Press.

13. García López, L. M., y Gutiérrez, D. (2013). The effects of a sport education season on empathy and assertiveness. Physical Education \& Sport Pedagogy, (i-first article). DOI: 10.1080/17408989.2013.780592.

14. Gutiérrez, D., García-López, L. M., Hastie, P. A., y Calderón-Luquin, A. (2013). The responses of Spanish students' to participation in seasons of sport education. The Global Journal of Health and Physical Education Pedagogy, 2(2), 111-127.

15. Hair, J. F., Anderson, R. E., Tatham, R. L., y Black, W. C. (1998). Multivariate Data Analisys. Upper Saddle River, NJ: Prentice Hall.

16. Hastie, P. A. (1996). Student role involvement during a unit of sport education. Journal of Teaching in Physical Education, 16, 88-103.

17. Hastie, P. A. (1998). Skill and tactical development during a sport education season. Research Quarterly for Exercise \& Sport, 69(4), 368-379.

18. Hastie, P. A., Martínez de Ojeda, D., y Calderón-Luquin, A. (2011). A review of research on Sport Education: 2004 to the present. Physical Education \& Sport Pedagogy, 16(2), 103-132. DOI:10.1080/17408989. 2010.535202.

19. Hastie, P.A., Sinelnikov, O.A. y Guarino. A.J. (2009). The development of skill and tactical competencies during a season of badminton. European Journal of Sport Science, 9(3), 133-40.

20. Hastie, P.A., Sinelnikov, O.A., Wallhead, T. y Layne, T. (2014). Perceived and actual motivational climate of a mastery-involving sport education season. European Physical Education Review, 20(2), 215-228.

21. Hastie, P. y Trost, S. G. (2002). Student physical activity levels during a Sport Education season. Pediatric Exercise Science, 14, 64-74.

22. Kinchin, G.D., Wardle, C., Roderick, S., y Sprosen, A. (2004). A survey of year 9 boys' perceptions of sport education in one English secondary school. Bulletin of Physical Education 40(1), 27-40.
23. Ko, B., Wallhead, T., y Ward, P. (2006). Chapter 4: Professional Development Workshops--What Do Teachers Learn and Use? Journal of Teaching in Physical Education, 25(4), 397-412.

24. MacPhail, A., Gorely, T., Kirk, D., y Kinchin, G. (2008). Children's experiences of fun and enjoyment during a season of sport education. Research Quarterly for Exercise and Sport, 79(3) 344-355.

25. Moller, A. C., y Elliot, A. J. (2006). The 2 X 2 achievement goal framework: An overview of empirical research. En A. Mittel (Ed.), Focus on educational psychology (pp. 307-326). New York: Nova Science Publishers, Inc.

26. Moreno, J. A., Cervelló, E. M., Montero, C. y Vera, J. A. (2012). Metas sociales, necesidades psicológicas básicas y motivación intrínseca como predictores de la percepción del esfuerzo en las clases de educación física. Revista de Psicología del Deporte, 21, 215-221.

27. Moreno, J. A., González-Cutre, D., Chillón, M., y Parra, N. (2008). Adaptación a la Educación física de la escala de las necesidades psicológicas básicas en el ejercicio. Revista Mexicana de Psicología, 25(2), 295-303.

28. Moreno, J. A., y Martínez, A. (2006). Importancia de la teoría de la autodeterminación en la práctica físico-deportiva: fundamentos e implicaciones prácticas. Cuadernos de Psicología del Deporte, 6(2), 39-54.

29. Niemec, C. P., y Ryan, R. M. (2009). Autonomy, competence, and relatedness in the classroom: Applying self-determination theory to de educational practice. Theory and Research in Education, 7(2), 133-144. DOI: $177 / 1477878509104318$.

30. Ntoumanis, N (2001). Step-by-step guide to SPSS for sport and exercise studies. Londres, UK: Routledge

31. Ntoumanis, N. (2005). A prospective study of participation in optional school physical education using a self-determination theory framework. Journal of Educational Psychology, 97, 444-453. DOI: 10.1037/0022-0663.97.3.444.

32. Papaioannou, C., Evaggelinou, C., Barkoukis, V., y Block, M. (2013). Disability awareness program in a summer camp. European Journal of Adapted Physical Activity, 6(2), 19-28.

33. Perlman, D. J. (2010). Change in affect and needs satisfaction for amotivated students within the sport education model. Journal of Teaching in Physical Education, 29(4), 433-45.

34. Perlman, D. J. (2011). Examination of self-determination within the sport education model. Asia-Pacific Journal of Health, Sport and Physical Education, 2, 79-92.

35. Pritchard, T., Hawkins, A., Wiegand, R., y Metzler, J. N. (2008). Effects of two instructional approaches on skill development, knowledge, and game performance. Measurement in Physical Education \& Exercise Science, 12(4), 219-236.

36. Ryan, R. M. y Deci, E. L. (2000). Self-determination theory and the facilitation of intrinsic motivation, social development, and well-being. American Psychologist, 55, 68-78. DOI: 10.1037/0003-066X.55.1.68

37. Ryan, R. M. y Deci, E. L. (2002). Overview of Self-determination theory: An organismic dialectical perspective. En E. L. Deci y R.M. Ryan (Eds.), Handbook of self-determination research (pp. 3-33). Rochester, NY: University of Rochester Press.

38. Sicilia, A. y Delgado, M. A. (2002). Educación Física y estilos de enseñanza. Barcelona, España: Inde.

39. Siedentop, D. (1994). Sport education: quality PE through positive sport experiences. Champaign, Ill.: Human Kinetics Publishers.

40. Siedentop, D., Hastie, P. A., y van der Mars, H. (2011). Complete Guide to Sport Education. Champaign, IL: Human Kinetics.

41. Sinelnikov, O. A. (2009). Sport Education for Teachers: Professional Development When Introducing a Novel Curriculum Model. European Physical Education Review, 15(1), 91-114. DOI: 10.1177/1356336X09105213. 
42. Sinelnikov, O., Hastie, y P. Prusak, K. (2007). Situational motivation in a season of sport education. ICHPER-SD Research Journal, 2, 43-47.

43. Spittle, M. y Byrne, K. (2009). The influence of sport education on student motivation in physical education. Physical Education and Sport Pedagogy, 14(3), 253-266.

44. Standage, M., Duda, J.L. y Ntoumanis, N. (2005). A test of self-determination theory in school physical education. British Journal of Educational Psychologym, 75, 411-433. DOI:10.1348/000709904X22359.

45. Vallerand, R.J. (1997). Toward a hierarchical model of intrinsic and extrinsic motivation. En M.P. Zanna (Ed.): Advances in experimental social psychology (pp. 271-360). New York: Academic Press.

46. Vlachopoulos, S. P. y Michailidou, S. (2006). Development and Initial Validation of a Measure of Autonomy, Competence, and Relatedness in Exercise: The Basic Psychological Needs in Exercise Scale. Measurement in Physical Education and Exercise Science, 10(3), 179-201.

47. Wallhead, T. L., Garn, A. C. y Vidoni, C. (2013). Sport Education and social goals in physical education: relationships with enjoyment, relatedness, and leisure-time physical activity. Physical Education and Sport Pedagogy, 18(4), 427-441. DOI:10.1080/17408989.2012.690377.

48. Wallhead, T.L., Hagger, M. y Smith, D. (2010). Sport education and extra-curricular sport participation: An examination using the transcontextual model of motivation. Research Quarterly for Exercise and Sport, 81, 442-455.

49. Wallhead, T. L. y Ntoumanis, N. (2004). Effects of a sport education intervention on students' motivational responses physical education. Journal of Teaching of Physical Education, 23, 4-18

50. Wallhead, T. L., y O'Sullivan, M. (2005). Sport Education: physical education for the new millennium? Physical Education \& Sport Pedagogy, 10(2), 181-210

51. Winer, B.J., Brown, D.R., y Michels, K.M. (1991). Statistical Principles in Experimental Design. New York: McGraw Hill. 